\title{
DIRECT NUMERICAL SIMULATIONS OF A HIGH PRESSURE TURBINE VANE
}

\author{
Andrew P. S. Wheeler, ${ }^{*}$, Richard D. Sandberg ${ }^{2}$, Neil D. Sandham² ${ }^{2}$ Richard Pichler $^{2}$, \\ Vittorio Michelassi ${ }^{3}$, and Greg Laskowski ${ }^{4}$ \\ ${ }^{1}$ Whittle Laboratory, University of Cambridge, Cambridge, UK \\ ${ }^{2}$ Engineering and the Environment, University of Southampton, Southampton, UK \\ ${ }^{3}$ GE Global Research, Munich, Germany \\ ${ }^{4}$ GE Aviation, Cincinnati OH, USA
}

\begin{abstract}
In this paper we establish a benchmark data set of a generic high-pressure turbine vane generated by direct numerical simulation (DNS) to resolve fully the flow. The test conditions for this case are a Reynolds number of 0.57 million and an exit Mach number of 0.9, which is representative of a modern transonic high-pressure turbine vane. In this study we first compare the simulation results with previously published experimental data. We then investigate how turbulence affects the surface flow physics and heat transfer. An analysis of the development of loss through the vane passage is also performed. The results indicate that free-stream turbulence tends to induce streaks within the near wall flow, which augment the surface heat transfer. Turbulent breakdown is observed over the late suction surface, and this occurs via the growth of two-dimensional Kelvin-Helmholtz spanwise roll-ups, which then develop into lambda vortices creating large local peaks in the surface heat transfer. Turbulent dissipation is found to significantly increase losses within the trailing-edge region of the vane.
\end{abstract}

\section{NOMENCLATURE}

C chord

e internal energy

$h \quad$ enthalpy

*Corresponding author Email: a.wheeler@eng.cam.ac.uk 
$k \quad$ conductivity

$l \quad$ passage height

M Mach number

p pressure

$q \quad$ surface heat flux

$R \quad$ gas constant

Re Reynolds number

$s \quad$ entropy

$T \quad$ Temperature

$T u \quad$ inlet turbulence intensity

$u \quad$ axial velocity

$v \quad$ tangential velocity

V total velocity

$w \quad$ spanwise velocity

$x \quad$ surface distance or axial distance

$y \quad$ wall normal distance or tangential distance

z $\quad$ spanwise distance

\section{Greek letters}

$\alpha \quad$ absolute flow angle

$\varepsilon \quad$ turbulent kinetic energy dissipation

$\gamma \quad$ ratio of specific heats

$\mu \quad$ dynamic viscosity

$\rho \quad$ density

\section{Subscripts}

$\begin{array}{ll}\text { ax } & \text { axial } \\ \text { ex } & \text { exit } \\ \text { in } & \text { inlet } \\ o & \text { stagnation condition }\end{array}$




\section{Superscripts}

$+\quad$ distance in wall units

\section{INTRODUCTION}

One of the greatest challenges to modern computational fluid dynamics is to fully simulate the turbulent flow within an aero-engine high-pressure turbine. This is because the flows in the high-pressure turbine operate at both transonic Mach numbers and high Reynolds numbers. There is also a strong interaction of stationary and rotating blades which creates a highly unsteady and non-uniform flow field that cannot be simulated correctly with current turbulence models. This is important because unsteadiness and turbulence affects both the aerodynamic efficiency and the heat transfer from the gas to the metal components; the life of the turbine blades is determined by the heat-load which is very sensitive to turbulent convection. Up to now, fully resolving the turbulence and unsteady flow field through Direct Numerical Simulation (DNS) has been prohibitively expensive due to the requirement to resolve the very large range of temporal and spatial scales in the flow within the high-pressure turbine. This paradigm is now beginning to change due to the development of massively parallelizable codes in combination with large-scale computing hardware, which is enabling the use of high-order direct simulation of turbulence at engine-scale conditions. Experimental measurements of turbulence properties within the high-pressure turbine are also very difficult to achieve due to the complexities of performing fast-response measurements within engine-scale experimental rigs. The lack of reliable modelling methods and experimental data is important because the development of more efficient aero-engines will only be possible if future high-pressure turbines can be designed with increased aerodynamic performance and durability as well as to withstand even hotter gas flows with reduced cooling. The drive for continued increases in turbine inlet temperatures means that the effects on heat transfer and performance of unsteady flows and turbulence will play an ever increasing role in the development of more efficient aircraft.

Early experimental work on transonic turbine cascades such as [1-3] showed that blade heat transfer is greatly affected by freestream turbulence and Mach number. These data also showed that despite the high Reynolds numbers in these flows, laminar boundarylayers can occur because of the strong favourable pressure gradients within the blade or vane passage [4]. This means that freestream turbulence plays a vital role on the surface heat transfer and the loss, because of its effect in promoting laminar/turbulent transition; both the boundary-layer loss and surface heat transfer are significantly increased by turbulence. Low speed rig and cascade data also showed that the effects of wake-induced unsteadiness augmented surface heat transfer on rotor blade surface [5-7]. Subsequent high-speed 
rotating rig experiments such as [8-12], revealed the incredible complexity of the flowfield within transonic high-pressure turbines, where the heat transfer and loss are affected by turbulence and unsteadiness generated by wakes and strong potential field interactions (such as trailing-edge shock interactions). Dunn [13] reviews much of this early work, including attempts to predict these flows using RANS modelling; these attempts often suffer from an inability for turbulence models to simulate accurately the effect of free-stream turbulence on the boundary-layer development and turbulent transition.

High pressure turbine vanes are likely to experience very high freestream turbulence levels due to the large-scale unsteadiness leaving the combustor. Experimental work at very high turbulence levels $(T u>10 \%)$, generally coupled with large turbulence length scales, show significant increases in surface heat flux and skin friction as compared to low intensity cases $(T u=2 \%)$ [14-16]. Interestingly, this work also shows that on the pressure-side the time-average boundary-layer profiles are typically laminar-like even at high turbulence intensities. The turbulence length-scale also affects heat transfer, and it has been shown that smaller length-scales tend to increase surface heat flux [17].

There are now a few examples of Large Eddy Simulations (LES) of HP turbine flows [18-20]. These show how the correct simulation of free-stream turbulence, and its decay, is crucial to determining the heat transfer on the blade surface (see [19]). This is particularly relevant to this paper, since they used the same experimental testcase for validation as used in this paper (i.e., the von Karman vane cascade [1]). They show near-wall streaks on the pressure-side, which they attribute to the formation of Görtler vortices, however the very low pressure-side curvature would suggest otherwise (indeed in the present paper we show that streaks are present on both the pressure-side and early suction-side, which have opposing curvatures, as discussed later). In any case, these near-wall streaks greatly augment the surface heat transfer but tend not to lead to turbulent breakdown. Direct numerical simulations (DNS) have up to now been limitied to low Mach number and Reynolds number turbine cases which are not directly applicable to transonic turbines [21-25].

In this paper we perform the first DNS of a high-pressure vane at transonic conditions to determine the true physical mechanisms which affect the vane heat transfer and loss. The test conditions and geometry are based on a previously published test-case [1]. The simulation conditions were performed at a Reynolds number of 0.57 million and an exit Mach number of 0.9 , which is representative of a modern transonic high-pressure turbine nozzle. In this study we first compare the simulation results with the previously published experimental data. We then investigate how turbulence affects the surface flow physics and heat transfer. An analysis of the development of loss through the vane passage is also performed, which is important from the perspective of HP turbine design.

\section{COMPUTATIONAL DETAILS}

The DNS were performed with a new in-house multi-block structured compressible Navier-Stokes solver purposely developed for exploiting high-performance computing systems [26]. The numerical method comprises a five-step, fourth-order accurate low- 
storage Runge-Kutta method [27], with a Fourier method for discretization of the spanwise direction. Additionally, a skew-symmetric splitting [28] is used to stabilize the convective terms. To date, DNS of compressible pipe flows [29], supersonic wakes [30] and turbulent jets in coflow [31] for analysis of jet noise have been performed with the code. The code has also been applied to low-pressure turbine flows [22] and transonic tip flows [32].

The code has also been used to study the effects of unsteadiness and turbulence on low-pressure turbine blade flows and has been validated against T106A low pressure (LP) turbine linear cascade measurements [22-25]. These LP turbine simulations were conducted by varying Reynolds number, flow coefficient and reduced frequency to investigate the steady and unsteady loss generation mechanism (such as profile and wake dilation losses) in a realistic design space.

Most quantities presented in this paper are non-dimensionalized: Lengths and distances are divided by the axial chord $\left(C_{a x}\right)$; and all other quantities are normalized by reference conditions, .

One of the objectives of this study is to compare the DNS calculations with previous experimental data. The testcase of Arts et al. [1] was used because this provided a detailed data set on a typical high pressure turbine vane geometry. The simulations were performed at conditions which matched conditions in this report, i.e. a vane exit Reynolds number of $\operatorname{Re}=(\rho V C / \mu)_{e x}=0.57 \times 10^{6}$, an exit Mach number of 0.9 and turbulence intensities at the inlet to the domain of $3.5-7 \%$. The published manufacturing coordinates shown in [1] were found to have unacceptable high frequency oscillations in the suface curvature distribution, and so the profile coordinates were re-interpolated using high order polynomial interpolation in order to ensure a smooth curvature distribution (see Figure 1). RANS calculations were performed for the original and modified profile to ensure there was no change in the loading distribution with this modification. The predicted loading distribution for the original and modified profiles are also shown in Figure 1, which demonstrates that the loading was not affected by the modification to surface curvature.

The solid boundaries on the vane were treated as a no-slip isothermal wall and the wall temperature was set to be $T_{w}=0.75 T_{o i n}$ to match the experimental data. It should be noted that for non-adiabatic CFD calculations it is common to use an isothermal wall condition, as performed in this study, although the true wall temperature will depend on the heat transfer to the solid as well as the fluid, which requires a conjugate analysis. The Nusselt number, however, is normally considered to be independent of wall temperature (provided the wall temperature does not greatly vary). Characteristic boundary-conditions were applied at the exit to avoid reflections from this boundary. A zonal characteristic boundary-condition [33] was applied over the final 95 streamwise grid lines, which has been previously shown to be highly effective in attenuating acoustic reflections from vortical structures passing through the outlet boundary.

Inlet turbulence was prescribed by the method of [24]; Perturbations are introduced in the three velocity components at the inlet using a sum of discrete waves. This is a computationally inexpensive method, but does lead to some coherence over the initial portion of the inlet domain (see Figure 2). The inlet turbulence level was set at 3.5 and $7 \%$. The calculated turbulence spectrum upstream of 
the vane with $T u=3.5 \%$ is shown in Figure 3. This shows that a broad range of scales enters the vane passage, however it should be noted that it was not possible to match the precise detail of the turbulence spectra observed in the experimental test-case; within the experimental wind tunnel integral length scales were measured to be about $30 \%$ of axial chord [34], while in the present simulation in order to maintain periodicity the maximum length-scale allowable within the spanwise extent of the domain was $5 \% C_{a x}$ and the integral length scale was estimated to be about $3 \%$ axial chord. Small turbulence length-scales lead to higher turbulent dissipation, and thus the difference in length-scale between these simulations and the experimental conditions means that the turbulence will decay much more rapidly in the simulations and the effect of this is discussed in more detail later. Also shown in Figure 3 is the turbulence spectrum within the wake, showing that the simulations capture a broad spectrum of turbulence energy over about 6 orders of magnitude.

Statistical quantities were determined using time-series data over 4 flow-through times. During the simulations, Favre (densityweighted) time-averaging was used to determine the time-mean flow field and turbulence quantities.

\section{Computational mesh and mesh sensitivity}

Meshes were created using a combination of different software. Initially a coarse grid was created using the Turbogrid software from ANSYS. This provided the block structure and block boundaries. A series of codes written in Matlab were then used to construct a much finer grid, ensuring optimum load balancing and near wall mesh control. The majority of the results described in this paper were obtained using a grid (Mesh A) which had a total of 2.5 million points in the blade-to-blade plane and 257 points in the spanwise plane (with 128 spanwise modes). Two other grids were tested: A coarser grid (Mesh B) which had roughly 1.2 million in-plane points and 193 spanwise points (96 modes); and a finer grid (Mesh C) with 3.6 million in-plane points and 257 spanwise points. Thus Mesh A had a total of 0.64 billion points, while Mesh B and C had 0.23 and 0.92 billion points respectively. Figure 4 shows the overall block structure and details of the mesh in the leading-edge and trailing-edge regions for Mesh A. In all cases, the spanwise extent was limited to $10 \%$ of the axial chord in order to limit computational costs. Figure 5 shows the spanwise mode amplitudes for Mesh A from monitor points in the transition region of the vane and in the wake; the figure shows a decay in mode amplitudes of over 2 orders of magnitude in velocity (i.e. 4 orders of magnitude in power) over the 128 modes simulated, demonstrating good spanwise resolution of the flow.

The sensitivity of the predicted wall shear stress to mesh density can be observed in Figure 6, which shows predictions of timeaverage wall shear stress for Mesh A, B and C. The results for the three meshes are in very close agreement over most of the vane surface. Differences between Mesh A (0.6 billion points) and Mesh C (0.92 billion points) are very slight. The coarsest grid (Mesh B, 0.23 billion points) shows some difference as compared to Meshes A and C close to the trailing-edge region on the suction-surface, and this is a result of a small difference in the prediction of the point of transition.

The near wall cell sizes are shown in Figure 7 for Mesh A. The average near wall grid spacings were $y^{+}=0.8, z^{+}=5, x^{+}=15$. 
Time-average boundary-layer profiles at various points on the vane surface are shown in Figure 8; typically there are around 125 points within the boundary-layer. The Kolmogorov length scale was determined from the time-average turbulent dissipation to be around $1.6 \times 10^{-4}$ of axial chord in the region of turbulent flow close to the vane trailing-edge, and this gave a near wall cell height of roughly 0.5 Kolmogorov length-scales. Figure 9 shows the ratio of the local cell size to the Kolmogorov length-scale computed from the local dissipation; within the wake, the cell sizes are typically less than 10 Kolmogorov length-scales. Previous DNS work (such as [35]) shows that the smallest scales in the flow are typically of order 10 Kolmogorov scales and grid independence is achieved when cell sizes are around 5-10 Kolmogorov lengths. Thus Mesh A is expected to be sufficiently well resolved to capture the smallest scales in the flow. Mesh A is used for all the following results described in this paper.

\section{COMPARISON WITH EXPERIMENTAL DATA}

In this section the computational results are compared with the data from Arts et al. [1]. Good agreement is observed when comparing the computational and experimental loading distributions (see Figure 10). Figure 11 shows experimental data on loss and exit angle, determined at $40 \%$ axial chord downstream of the trailing-edge, compared with the computational results. In order to be consistent with the experimental data, the loss coefficient $\left(\zeta_{2}\right)$ was defined in the same way as Arts et al. [1]

$$
\zeta_{2}=1-\frac{1-\left(\frac{p_{2}}{p_{o 2}}\right)^{\frac{\gamma-1}{\gamma}}}{1-\left(\frac{p_{2}}{p_{o 1}}\right)^{\frac{\gamma-1}{\gamma}}}
$$

where $p_{o 1}$ and $p_{o 2}$ are area-average inlet and exit total pressures, and $p_{2}$ is the area-average exit static pressure. Area-averaging of the total pressures was performed in order to be consistent with the experimental measurements, which were also area-averaged. The computational results sit within the scatter of the experimental data for $R e=0.5 M$; the quoted uncertainties in loss and angle measurement were 0.2 points and $0.5 \mathrm{deg}$ respectively [1]. It should also be noted that the computational results were obtained at a turbulence level of $3.5 \%$, but the published experimental results on loss were obtained at $1 \%$ turbulence level, although despite this the agreement between the predicted loss and the experimental data is very close.

A comparison of the experimentally measured heat flux with the predictions is shown in Figure 12. The experimental data is obtained over a range of turbulence levels $(T u=1,4,6 \%)$. The simulations were run with $T u=3.5$ and $7 \%$ at the inlet. It should be noted that (for reasons discussed above) the inflow turbulent length-scale was around a factor of ten smaller in the simulations as compared to the experimental conditions, and thus the decay of turbulence in the simulations is expected to be much greater than in the experiments. Figure 12 shows that in both the experimental data and simulations, increasing turbulence intensity tends to augment the heat transfer near the leading-edge on the pressure-surface and suction-surface. However the extent to which free-stream turbulence augments the heat transfer in these regions is larger in the experimental data, as compared to the simulations; this is most likely a result 
of the difference in turbulence decay rates between the DNS and experiments and this would also explain the lower heat transfer level observed in the DNS in this region.

Further aft on the suction-surface, the influence of turbulence level observed in the experimental data is isolated to the region of transitional flow $\left(x>0.9 C_{a x}\right)$, where increasing turbulence level brings about an earlier onset to turbulence. The computational simulations show a later onset of transition on the suction surface as compared to the experimental data at the same turbulence level. Again, this may be a result of differences in the turbulence spectra. Another uncertainty is the surface-curvature; it was mentioned earlier that the published coordinates showed undesirable fluctuations in surface curvature that were corrected in this study (see Figure 1), and these are particularly evident in the aft suction surface. It is not clear whether the manufactured profiles used in the experiment could also have curvature discontinuties, but this would indeed lead to earlier onset transition to turbulence if this were the case.

\section{TIME RESOLVED FLOW PHYSICS}

Figure 13 shows two typical snapshots of the simulated flowfield spaced $12 \%$ of a flow-through time apart, with a grey scale showing density gradient magnitude and colour scale showing near wall temperature. The figure shows the development of pressure waves (labelled 'a') which are generated by the intense vortex shedding which occurs at the trailing-edge. The pressure waves move upstream, affecting the aft suction surface. Following the passing of these waves, oscillations in the near wall gas temperature (indicative of surface heat flux) appear close to the trailing-edge, due to the development of two-dimensional Kelvin-Helmholtz structures (labelled 'b'); the boundary-layer profile in this region is inflexional (see Figure 8) and unstable. The structures eventually breakdown to turbulence as observed by the large increase in near wall gas temperature near the trailing-edge. Figure 13 also shows that pressure waves from the adjacent trailing-edge, which tend to propagate along the throat, impinge on the mid suction surface, causing local increases in near-wall gas temperature, and thus heat flux (labelled 'c').

Data from near-surface monitor points at a single spanwise location within the transition region on the suction surface were used to construct space-time diagrams of the evolution of the instability waves that develop in this region. These are shown in Figure 14. The results show that instability waves propagate from within a region of low shear stress at around $40 \%$ of the boundary-layer edge velocity. They develop and breakdown into turbulence, generating large fluctions in shear stress, heat flux and pressure. It can also be observed that the position at which these instabilities develop from fluctuates over time, and instability waves are not always present, but exist only over intermittent periods of time. Thus the location of turbulent transition moves considerably over the vane surface with time.

Further detail of the structure of these instabilities can be observed in Figure 15, which shows iso-surfaces of Q-criterion within this transition region at a typical instant in time. The iso-surfaces identify spanwise vortices which give rise to the heat flux signatures observed in Figures 13 and 14. Also shown in Figure 15 are the effect of shocks which create small scale structures within the planes 
normal to the flow direction. Where these shocks impinge on the suction surface, additional spanwise vortex structures are observed. These pressure waves move upstream over the suction surface at about $30 \%$ of the freestream velocity, and since the local flow Mach number in this region is around 0.95 , the Mach number relative to the pressure waves is about 1.2 ; the compression waves moving upstream from the trailing-edge therefore experience a supersonic flow relative to their own motion and so they rapidly coalesce to form shock waves.

Figure 16 is also a snapshot of the flow structure within the region of transition, showing a typical lambda-type vortex structure just prior to turbulent breakdown. These structures are sometimes observed in the late stages of transition (see for instance [36]).

Power spectra of the velocities in the near wall region shortly upstream of fully turbulent region are shown in Figure 17. Also shown in this figure is the spectrum for a monitor point in the wake, just downstream of the trailing-edge. The spectra upstream of turbulent breakdown show quite a broad peak, indicating that the two-dimensional instabilities have a relatively broad frequency content; the frequency of the instabilities is determined by the inviscid mechanism of instabilities which will grow within an inflexional profile.

On the pressure surface, Figure 18 shows long streamwise streaks in surface heat flux which extend all the way from the leadingedge to the trailing-edge (also shown in Figure 13 labelled 'd'). These streaks affect both the leading-edge region of the suction-surface and the pressure-surface, and thus are not a result of concave curvature effects and Görtler vortices as suggested by [19]. Figure 18 shows that the intensity of these streaks increases as the turbulence level is raised from 3.5 to $7 \%$, and thus the augmentation of the surface heat flux near the leading-edge as turbulence level is increased (as observed in Figure 12) is likely to be due to the influence of free-stream turbulence on these near wall streaks.

Near-wall streaks are a known consequence of the perturbation of freestream turbulence on laminar boundary-layers (see [37, 38]). Figure 19 shows how the freestream turbulence is distorted and stretched around the leading-edge, generating long streaks in the freestream. These are likely to induce the long streaks observed in the near-wall heat flux, since the free-stream streamwise vorticity will induce opposing vorticity near the wall.

\section{DEVELOPMENT OF LOSS}

Denton [39] demonstrates that turbomachinery losses can be directly computed from the rise in entropy due to irreversible processes. The rate of change of entropy within a control volume (C.V.) can be determined based on the entropy and heat fluxes through the control volume surfaces (C.S.) and the integral of internal entropy production within the volume as follows:

$$
\frac{\partial}{\partial t} \int_{C . V} \rho s d V=\int_{C . V} \theta d V-\int_{C . S} \rho s \mathbf{V} \bullet d \mathbf{A}-\int_{C . S .} \frac{\mathbf{q}}{T} \bullet d \mathbf{A}
$$


where $\theta$ is equal to the sum of the viscous dissipation and the effects of internal irreversible heat transfer:

$$
\theta=\frac{(\tau \bullet \nabla) \mathbf{V}}{T}+\frac{(\mathbf{q} \bullet \mathbf{q})}{k T^{2}}
$$

If equation 2 is integrated over sufficiently long times such that the flow is statistically stationary, the temporal derivative is eliminated and we can re-write equation 2 using time-average quantities denoted by an overline as follows:

$$
\int_{C . V} \bar{\theta} d V=\int_{C . S} \overline{\rho s \mathbf{V}} \bullet d \mathbf{A}+\int_{C . S} \overline{\left(\frac{\mathbf{q}}{T}\right)} \bullet d \mathbf{A}
$$

The loss sources are therefore contained within $\bar{\theta}$. The irreversible entropy generation rate per unit volume $(\theta)$ contains contributions from both irreversible heat transfer within the fluid and viscous dissipation. The viscous dissipation can be considered as being made up of a contribution from the mean velocity and strain field, and also the effects of unsteadiness, the latter being equal to the turbulent kinetic energy dissipation, thus:

$$
\bar{\theta}=\overline{\frac{\Phi}{T}+\frac{\varepsilon}{T}+\frac{\zeta}{T}},
$$

where $\Phi$ is the dissipation computed from the time-mean strain field, $\varepsilon$ is the turbulent kinetic energy dissipation and $\zeta$ is the contribution due to irreversible heat transfer $(\zeta=\mathbf{q} \bullet \mathbf{q} / k T)$. The time average of $\theta$ was determined by computing the instantaneous total dissipation terms $\left(\frac{\Phi}{T}+\frac{\varepsilon}{T}\right)$ and heat flux term $\left(\frac{\zeta}{T}\right)$ at each time-step, and then summing these over time. The contribution to entropy generation from the time-mean strain was then computed from the Favre-average flow, and this was then subtracted from the total dissipation term to obtain the contribution due to turbulent dissipation.

The rise in irreversible entropy production through the vane is plotted in Figure 20. This is computed by integrating over the volume between the inlet axial plane and a downstream axial plane, the contributions to irreversible entropy production.

Over the vane surfaces, the majority of the loss occurs due to the time-average strain field, since turbulent flow is only observed over the aft suction-surface and there is little turbulent dissipation before the trailing-edge. A large amount of the total loss is incurred within the trailing-edge region, where the majority of the dissipation results from the development of turbulent flows within this region. The intense mixing that occurs within the trailing-edge region leads to a large rise in dissipation. The turbulent dissipation downstream of the trailing-edge is far greater than the laminar dissipation, and thus the growth in loss due to turbulent dissipation is much larger than that observed from the time-average-strain dissipation downstream of the trailing-edge, where the flow is highly turbulent. At $x=2$ the contributions to the total from the time-mean strain, the turbulent dissipation and irreversible heat transfer are $40 \%, 40 \%$, and $20 \%$ respectively. 


\section{CONCLUSION}

In this paper we discussed the first DNS of a high-pressure vane at transonic conditions. The results show that the pressure surface has no clear transition to turbulence, but instead freestream turbulence induces near-wall streaks which augment the surface heat transfer. On the suction surface, transition to turbulence occurs near the vane trailing-edge and is predominantly caused by the intermittent development of Kelvin-Helmholtz roll-ups that become distorted into lambda vortices, before finally breaking down to turbulence. The position of transition was also found to vary significantly over extended periods of time. The flow within the trailing-edge region was dominated by highly turbulent vortex shedding, with a broad turbulence spectrum. Pressure waves, generated at the vane trailing-edge, were observed to propagate upstream and trigger the onset of these Kelvin-Helmholtz structures

The total contributions to entropy production from the time-mean strain, turbulent dissipation and irreversible heat transfer were determined; these were found to contribute roughly $40 \%, 40 \%$, and $20 \%$ to the total irreversible entropy generation respectively. The majority of turbulent dissipation was found to be generated within the trailing-edge region. The results of this loss analysis highlight the importance of the trailing edge flow in high-pressure turbines.

\section{ACKNOWLEDGMENT}

The authors gratefully acknowledge the support of the Partnership for Advanced Computing in Europe (PRACE) and the UK Turbulence Consortium funded by the EPSRC under grant number EP/L000261/1 for the computing resources used for this work.

\section{REFERENCES}

[1] Arts, T., Lambert, R., and Rutherford, A. W., 1990. "Aero-thermal investigation of a highly loaded transonic linear turbne guide vane cascade". von Karman Institute Technical Note 174.

[2] Nicholson, J. H., Forest, A. E., Oldfield, M. L. G., and Schultz, D. L., 1984. "Heat transfer optimized turbine rotor blades- an experimental study using transient techniques". Journal of Engineering for Gas Turbines and Power, 106, January.

[3] Consigny, H., and Richards, B. E., 1982. "Short duration measurements of heat transfer rate to a gas turbine rotor blade". Journal of Engineering for Power, 104, July.

[4] Mayle, R. E., 1991. "The role of laminar-turbulent transition in gas turbine engines". Journal of Turbomachinery, 113, October, pp. 509-537.

[5] Dring, R. P., Blair, M. F., Joslyn, H. D., Power, G. D., and Verdon, J. M., 1990. "The effects of inlet turbulence and rotor/stator interactions on aerodynamics and heat transfer of a large-scale rotating turbine model". Journal of Turbomachinery, 112, April. 
[6] Hodson, H. P., 1984. "Boundary layer and loss measurements on the rotor of an axial flow turbine". Journal of Engineering for Gas Turbines and Power, 106, April.

[7] Mayle, R. E., and Dullenkopf, K., 1990. “A theory for wake induced transition”. Journal of Turbomachinery, 112, April.

[8] Allan, W. D., Ainsworth, R., and Thorpe, S., 2008. "Unsteady heat transfer measurements from transonic turbine blades at engine representative conditions in a transient facility". Journal of Engineering for Gas Turbines and Power, 130(4), July, p. 041901.

[9] Didier, F., Dénos, R., and Arts, T., 2002. "Unsteady rotor heat transfer in a transonic turbine stage". Journal of Turbomachinery, 124, October, pp. 614-622.

[10] Haldeman, C. W., and Dunn, M. G., 2004. "Heat-transfer measurements and predictions for the vane and blade of a rotating high-pressure turbine stage". Journal of Turbomachinery, 126, January, pp. 101-109.

[11] Haldeman, C. W., Dunn, M. G., Barter, J. W., Green, B. R., and Bergholz, R. F., 2005. “Aerodynamic and heat-flux measurements with predictions on a modern one and one-half state high pressure transonic turbine". Journal of Turbomachinery, 127, July, pp. 522-523.

[12] Guenette, G. R., Epstein, A. H., Giles, M. B., Haimes, R., and Norton, R. J. G., 1989. "Fully scaled transonic turbine rotor heat transfer measurements". Journal of Turbomachinery, 111, January, pp. 1-7.

[13] Dunn, M. G., 2001. “Convective heat transder and aerodynamics in axial flow turbines”. Journal of Turbomachinery, 123, October.

[14] Radomsky, R. W., and Thole, K. A., 2000. "Flowfield measurements for a highly turbulent flow in a stator vane passage". Journal of Turbomachinery, 122, April.

[15] Radomsky, R. W., and Thole, K. A., 2002. "Detailed boundary layer measurements on a turbine stator vane at elevated freestream turbulence levels". Journal of Turbomachinery, 124, January.

[16] Nasir, S., Carullo, J. S., Ng, W. F., Thole, K. A., Wu, H., Zhang, L. J., and Moon, H. K., 2009. "Effects of large scale high freestream turbulence and exit reynolds number on turbine vane heat transfer in a transonic cascade". Journal of Turbomachinery, 131, April.

[17] Holmberg, D. G., and Diller, T. E., 2005. "Simultaneous heat flux and velocity measurements in a transonic turbine cascade". Journal of Turbomachinery, 127, July, pp. 502-506.

[18] Fransen, R., Collado, E., Duchaine, F., Gourdain, N., Gicquel, L., Vial, L., and Bonneau, G. "Comparison of RANS and LES in high pressure turbines". In 3me Colloque INCA, ONERA Toulouse, November 17-18 2011.

[19] Gourdain, N., Gicquel, L., and Collado, E., 2012. "Comparison of RANS and LES for prediction of wall heat transfer in a highly loaded turbine guide vane". Journal of Propulsion and Power, 28, pp. 423-433.

[20] Gourdain, N., Gicquel, L., Fransen, R., Collado, E., and Arts, T., 2011. "Application of RANS and LES to the prediction of flows 
in high pressure turbine components". ASME Paper GT2011-46518.

[21] Wissink, J., and Rodi, W., 2006. "Direct numerical simulation of flow and heat transfer in a turbine cascade with incoming wakes". Journal of Fluid Mechanics, pp. 209-247.

[22] Sandberg, R., Pichler, R., and Chen, L., 2012. "Assessing the sensitivity of turbine cascade flow to inflow disturbances using direct numerical simulation.”. In ISUAAAT-13 (2012): The 13th International Symposium on Unsteady Aerodynamics, Aeroacoustics and Aeroelasticity of Turbomachines.

[23] Chen, L., Pichler, R., and Sandberg, R. D., 2013. "Compressible dns of a low pressure turbine subjected to inlet disturbances". In DLES9: Workshop on Direct and Large-Eddy Simulation, 03-05 April 2013., Dresden, DE, 03 - 05 Apr 2013.

[24] Sandberg, R. D., Pichler, R., Chen, L., Johnstone, R., and Michelassi, V. "Compressible Direct Numerical Simulation of lowpressure turbines: Part I Methodology”. In ASME Turbo Expo 2014, ASME Paper no. GT2014-25685 (Accepted for publication in the Journal of Turbomachinery).

[25] Michelassi, V., Sandberg, R. D., Pichler, R., and Chen, L. “Compressible Direct Numerical Simulation of low-pressure turbines: Part II Effect of inflow disturbances”. In ASME Turbo Expo 2014, ASME Paper no. GT2014-25689 (Accepted for publication in the Journal of Turbomachinery).

[26] "Cray centre of excellence project report".

[27] Kennedy, C., Carpenter, M., and Lewis, R., 2000. "Low-storage, explicit runge-kutta schemes for the compressible navier-stokes equations". Applied Numerical Mathematics, 35(3).

[28] Kennedy, C., and Gruber, A., 2008. "Reduced aliasing formulations of the convective terms within the navier-stokes equations for a compressible fluid". Journal of Computational Physics, 227(3).

[29] Sandberg, R. D., Suponitsky, V., and Sandham, N. D., 2010. "DNS of a canonical nozzle flow". proceedings of the ERCOFTAC Workshop Direct and Large-Eddy Simulations 8.

[30] Sandberg, R. D., 2012. "Numerical investigation of turbulent supersonic axisymmetric wakes". Journal of Fluid Mechanics, 702, 6, pp. 488-520.

[31] Sandberg, R. D., Sandham, N., and Suponitsky, V., 2012. "DNS of compressible pipe flow exiting into a coflow". International Journal of Heat and Fluid Flow, 35, pp. $33-44$.

[32] Wheeler, A. P. S., and Sandberg, R. D., 2012. "Direct numerical simulation of a transonic turbine tip flow". In ISUAAAT-13 (2012): The 13th International Symposium on Unsteady Aerodynamics, Aeroacoustics and Aeroelasticity of Turbomachines.

[33] Sandberg, R. D., and Sandham, N. D., 2006. "Nonreflecting zonal characteristic boundary condition for direct numerical simulation of aerodynamic sound”. AIAA Journal, 44(2). 
[34] Arts, T., 2014. "private communication".

[35] Yao, Y., Thomas, T., and Sandham, N. D., 2001. "Direct numerical simulation of turbulent flow over a rectangular trailingedge". Theoretical and Computational Fluid Dynamics, pp. 337-358.

[36] Alam, M., and Sandham, N. D., 2000. "Direct numerical simulation of 'short' laminar separation bubbles with turbulent reattachment”. Journal of Fluid Mechanics, pp. 223-249.

[37] Jacobs, R. G., and Durbin, P. A., 2001. "Simulations of bypass transition”. Journal of Fluid Mechanics, 428, pp. 185-212.

[38] Anthony, R. J., Jones, T. V., and LaGraff, J. E., 2005. "High frequency surface heat flux imaging of bypass transition". Journal of Turbomachinery, 127, April.

[39] Denton, J. D., 1993. “Loss mechanisms in turbomachines”. Journal of Turbomachinery, 115, pp. 621-656.

\section{Figures}

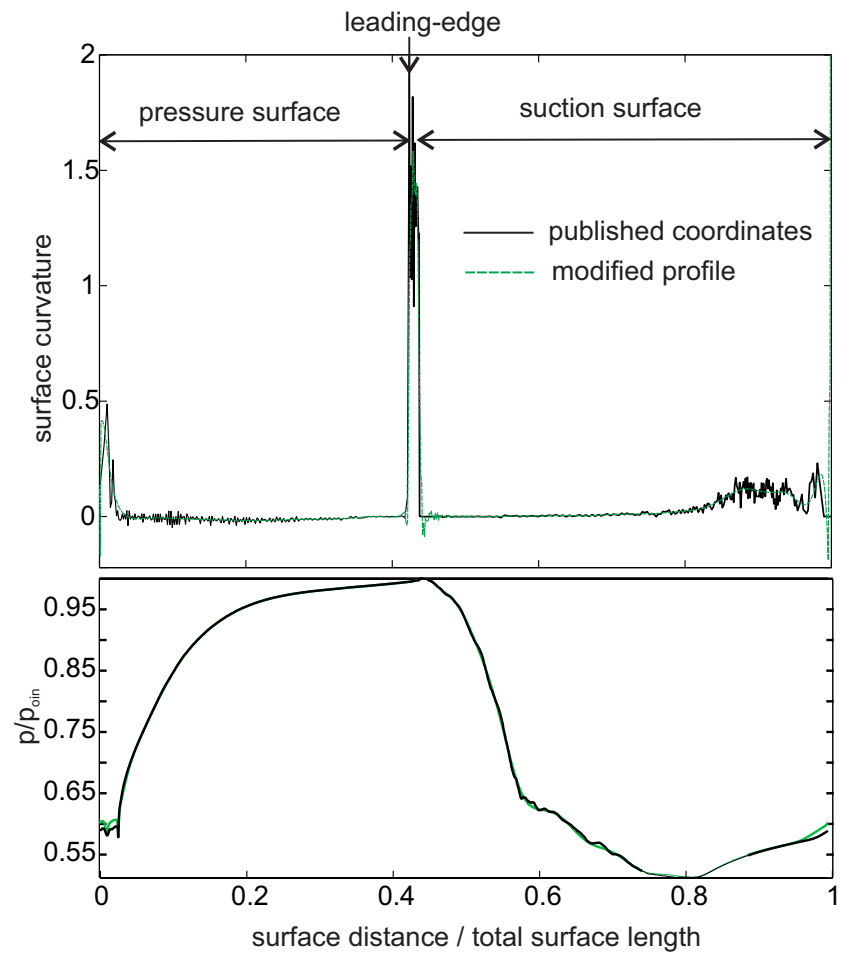

FIGURE 1. Surface curvature of vane determined from published manufacturing coordinates of [1] and the corrected profile used in this study 


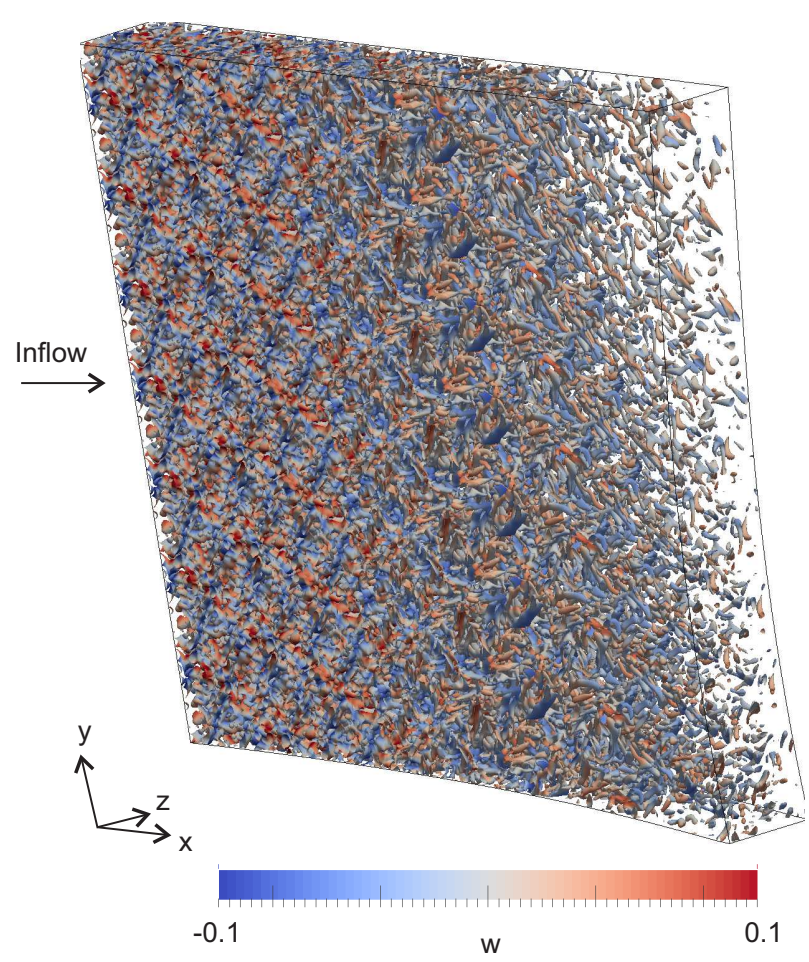

FIGURE 2. Snapshot of isosurface of Q-criterion $=25$ with inlet free-stream turbulence $T u=3.5 \%$. Isosurface colored with spanwise velocity $w$ normalized by the inlet velocity (block 2 shown)
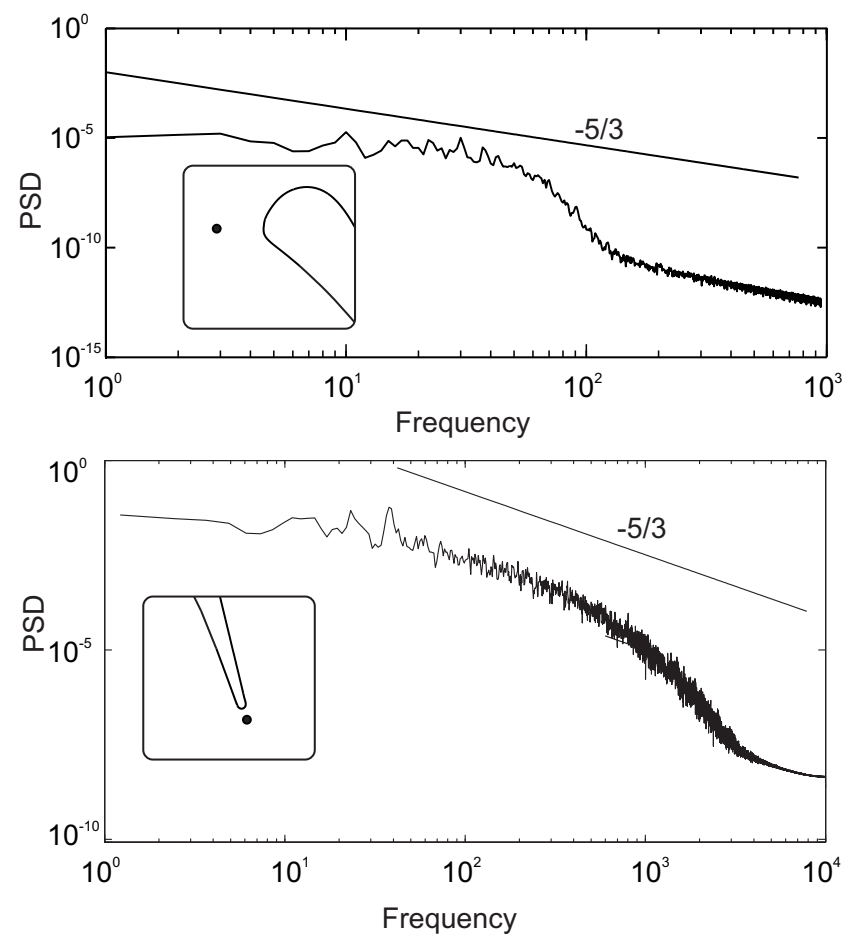

FIGURE 3. Power spectral density of total velocity upstream of the vane leading edge $(x=-0.3, y=0.0)$ and within the wake $(x=1.017, y=-1.49)$ 


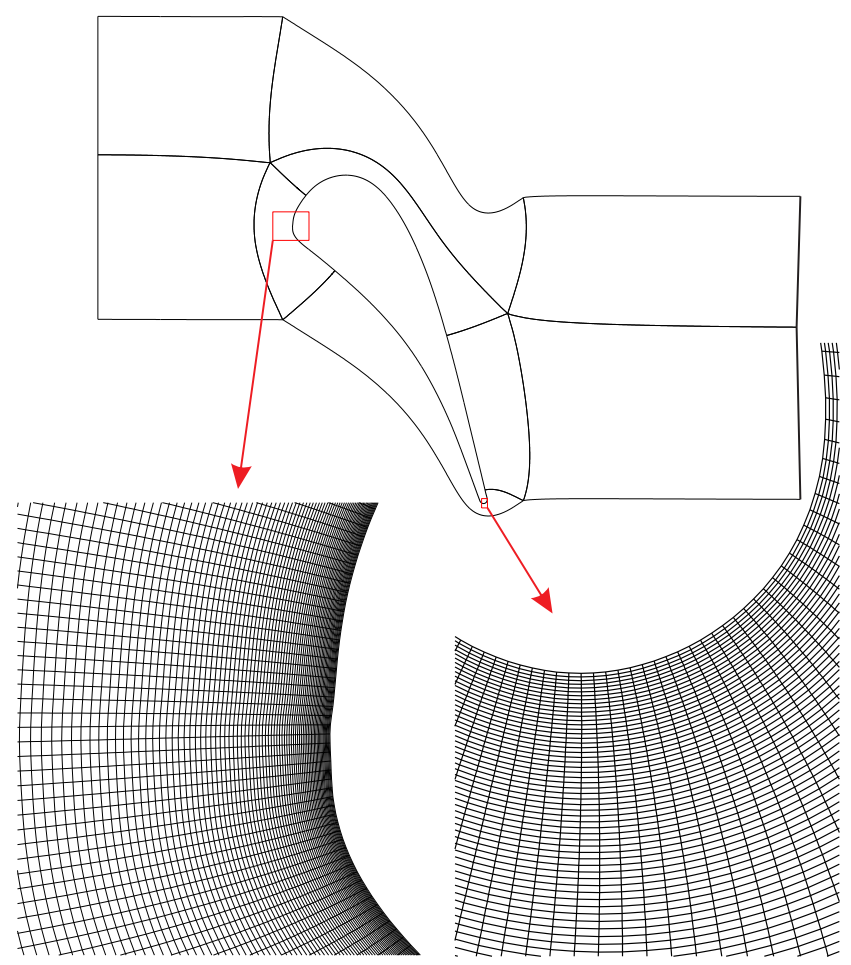

FIGURE 4. Mesh block structure and mesh details at the leading and trailing edges. Every $4^{\text {th }}$ grid line is shown (Mesh A)

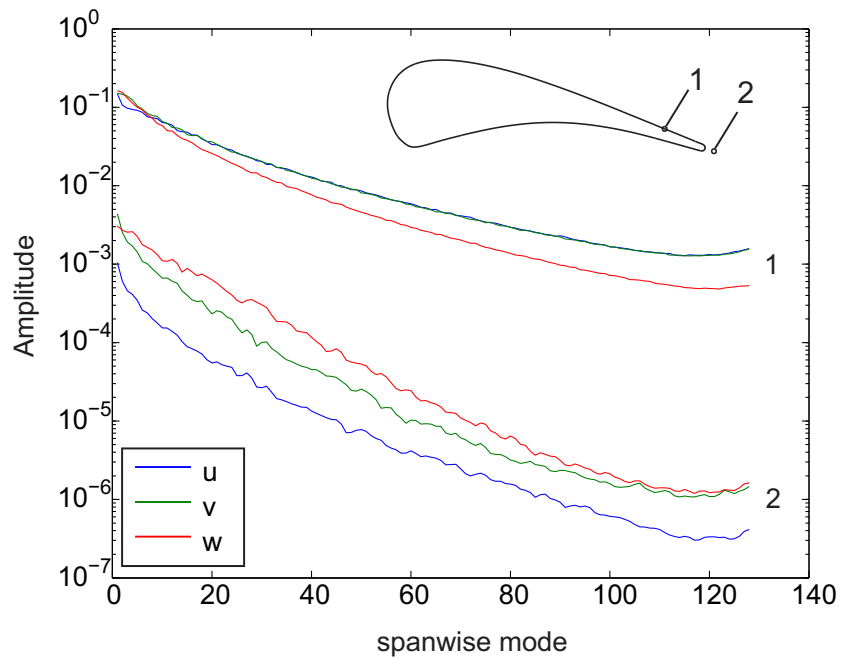

FIGURE 5. Spanwise mode amplitudes (Mesh A) 


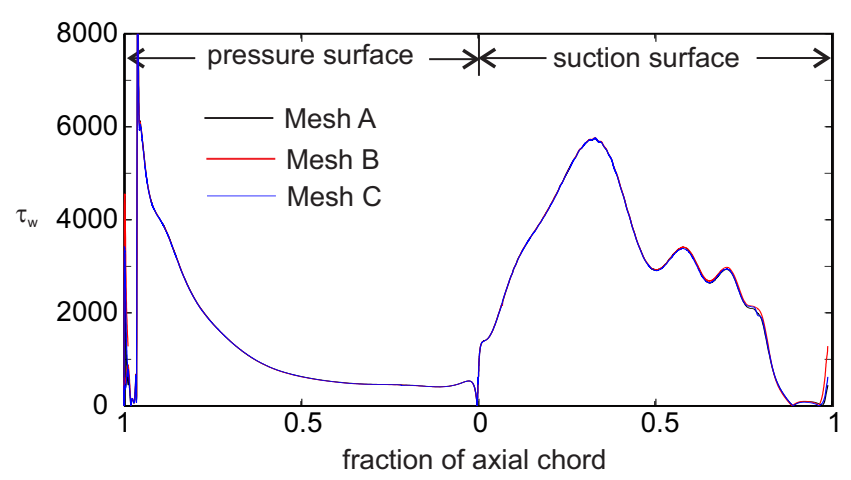

FIGURE 6. Sensitivity of wall shear stress to mesh density

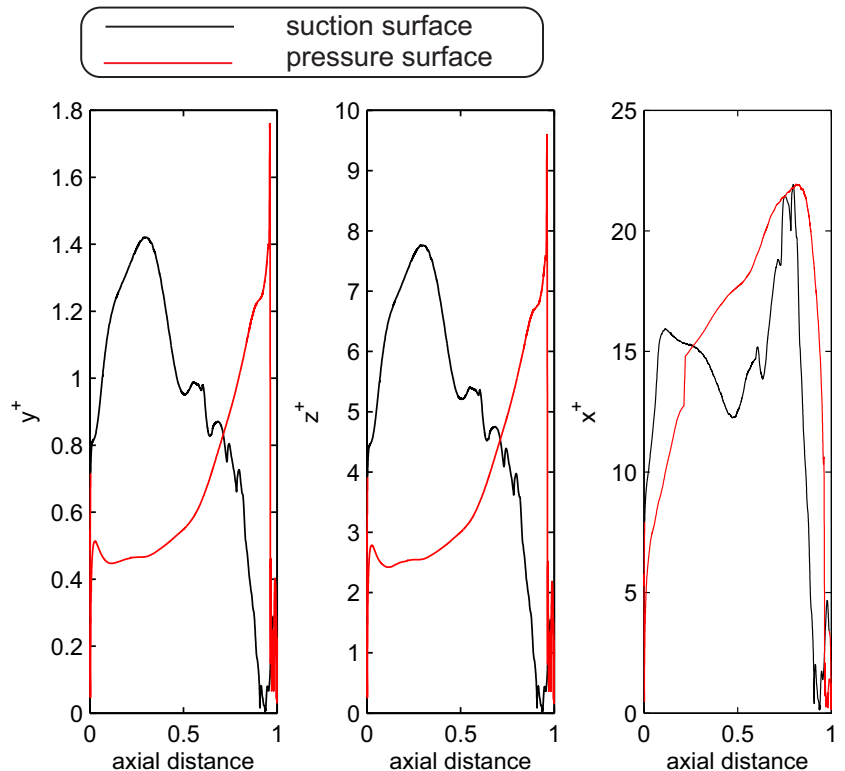

FIGURE 7. Near wall grid size measured in wall units (Mesh A)
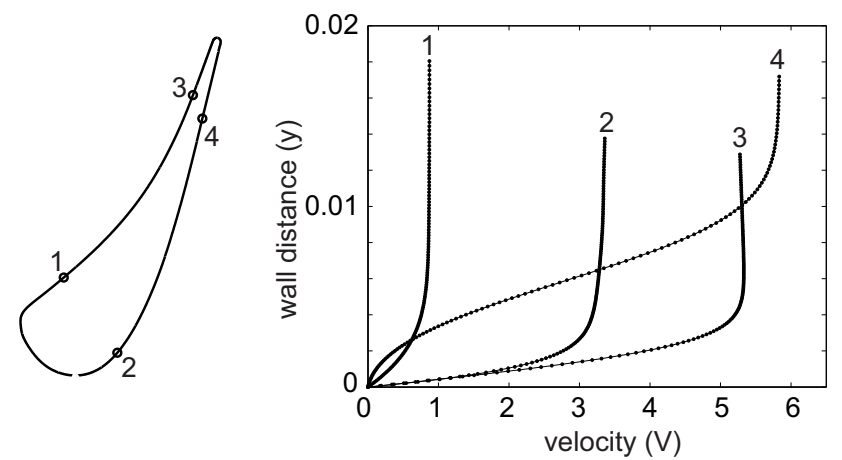

FIGURE 8. Boundary-layer time-average velocity profiles, 125 grid points shown (Mesh A) 


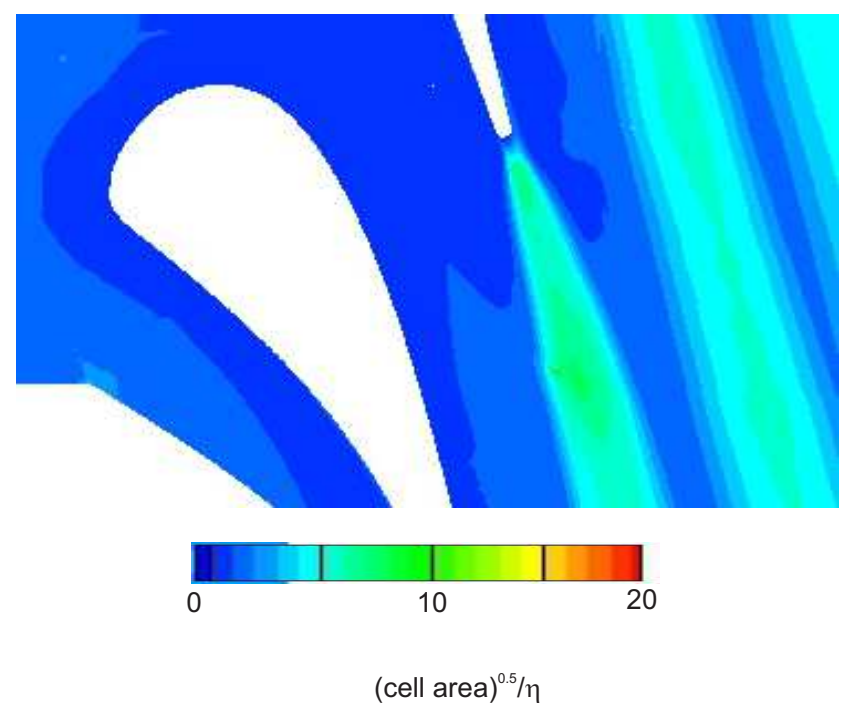

FIGURE 9. Ratio of in-plane cell size to Kolmogorov length-scale computed from time average turbulent dissipation for Mesh A

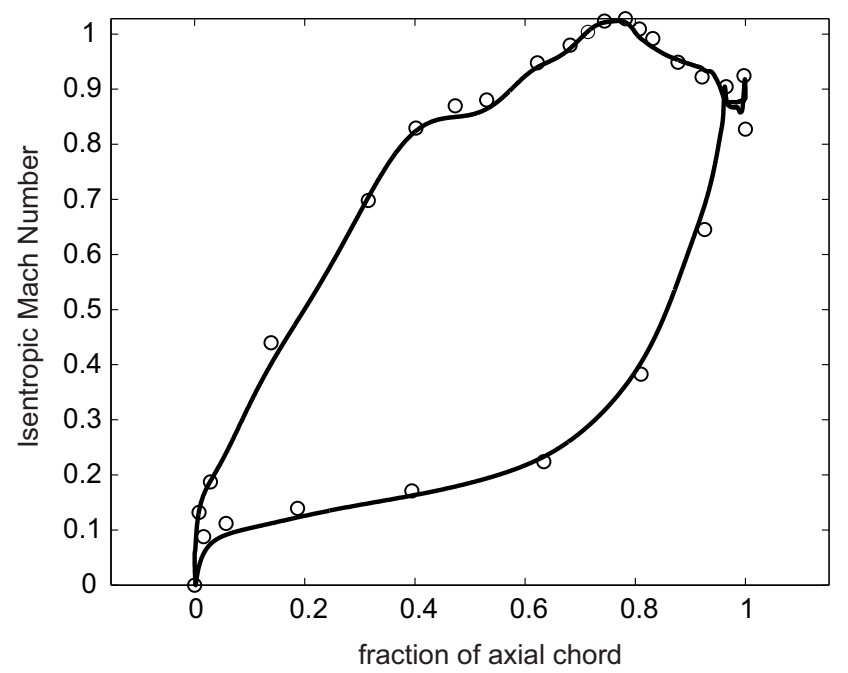

FIGURE 10. Isentropic Mach number distribution and comparison with experimental data of Arts et al. [1] 


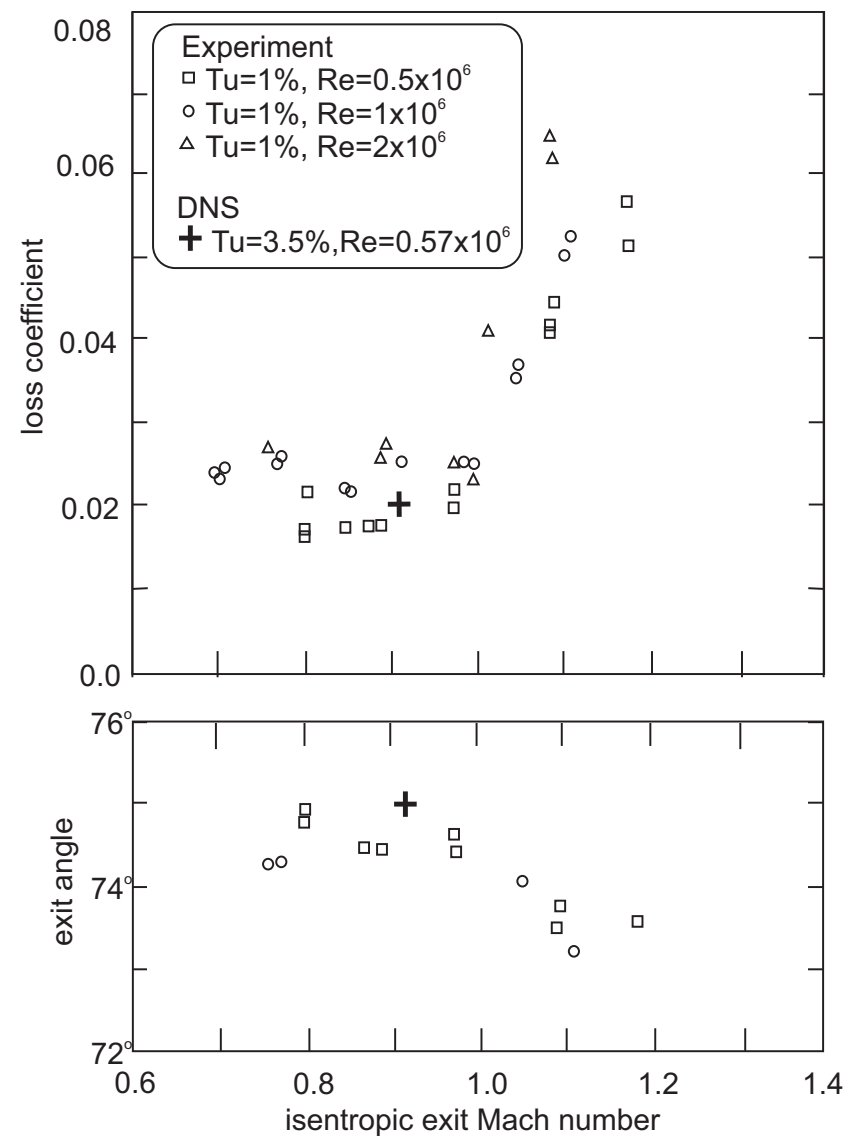

FIGURE 11. Comparison of time-average loss and exit angle with experimental data of Arts et al. [1] determined at $40 \% C_{a x}$ axial chord downstream of the trailing-edge.

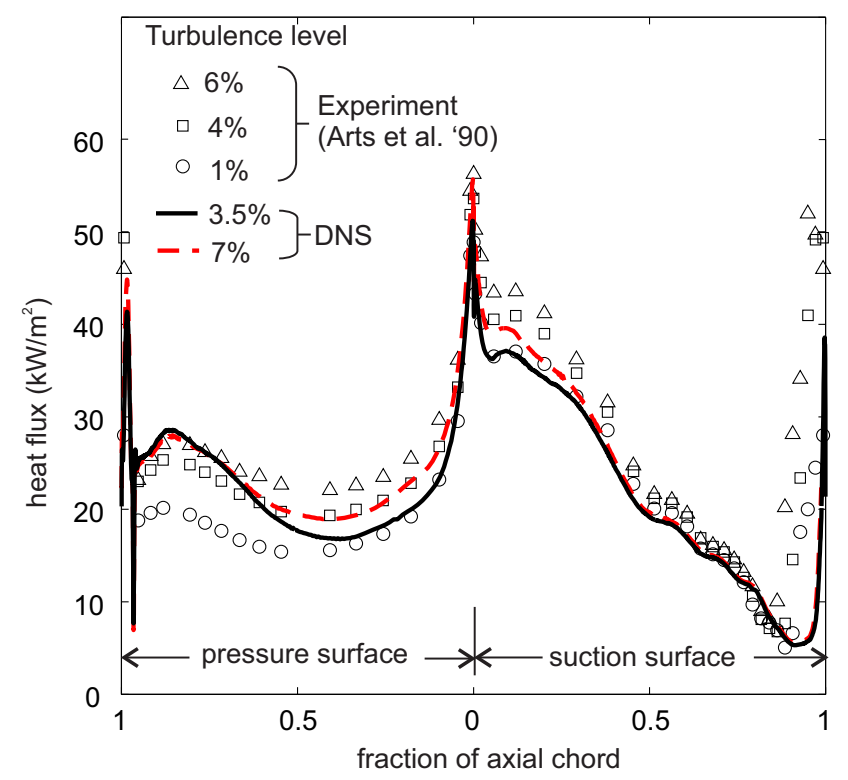

FIGURE 12. Comparison of predicted heat flux with experimental data of Arts et al. [1] 


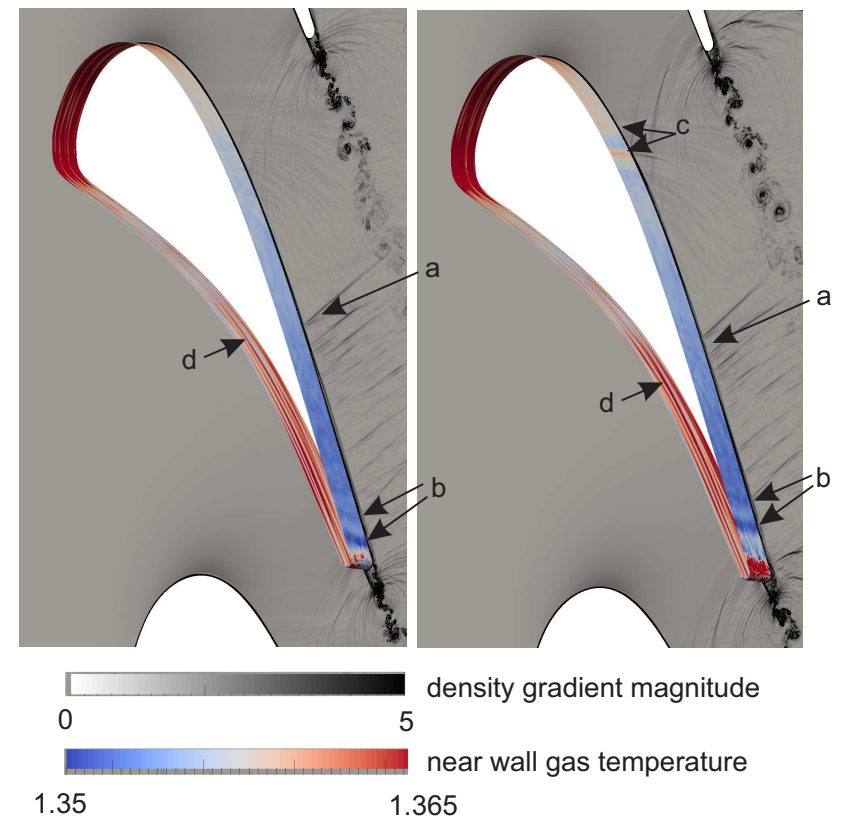

FIGURE 13. Two instantaneous snapshots $(\Delta t=0.12)$ showing contours of density gradient magnitude (greyscale) and gas temperature at the first grid point away from the wall (colourscale). Marked on the figure are: (a) upstream moving pressure waves; (b) development of $2 d$ spanwise instabilities over the aft suction-surface; (c) fluctuations in surface heat flux due to pressure wave reflections ;(d) near wall streaks.

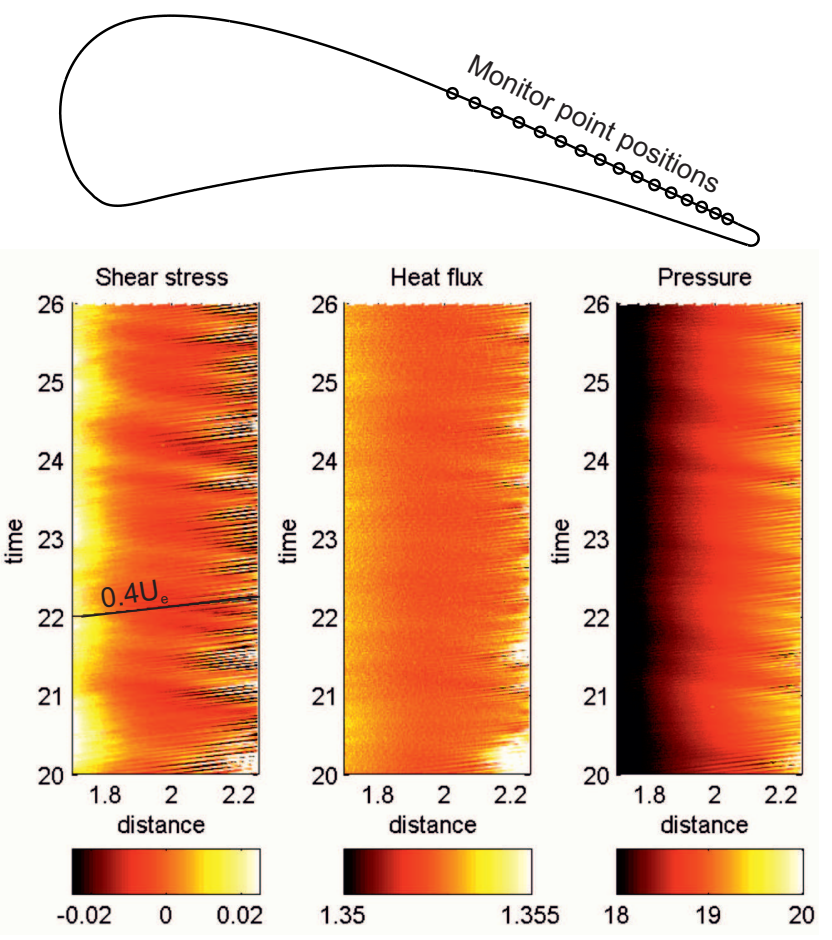

FIGURE 14. Space-time diagrams of surface shear, heat flux and pressure from monitor points over the aft suction surface. The surface distance is measured from the leading-edge, and normalized by axial chord 


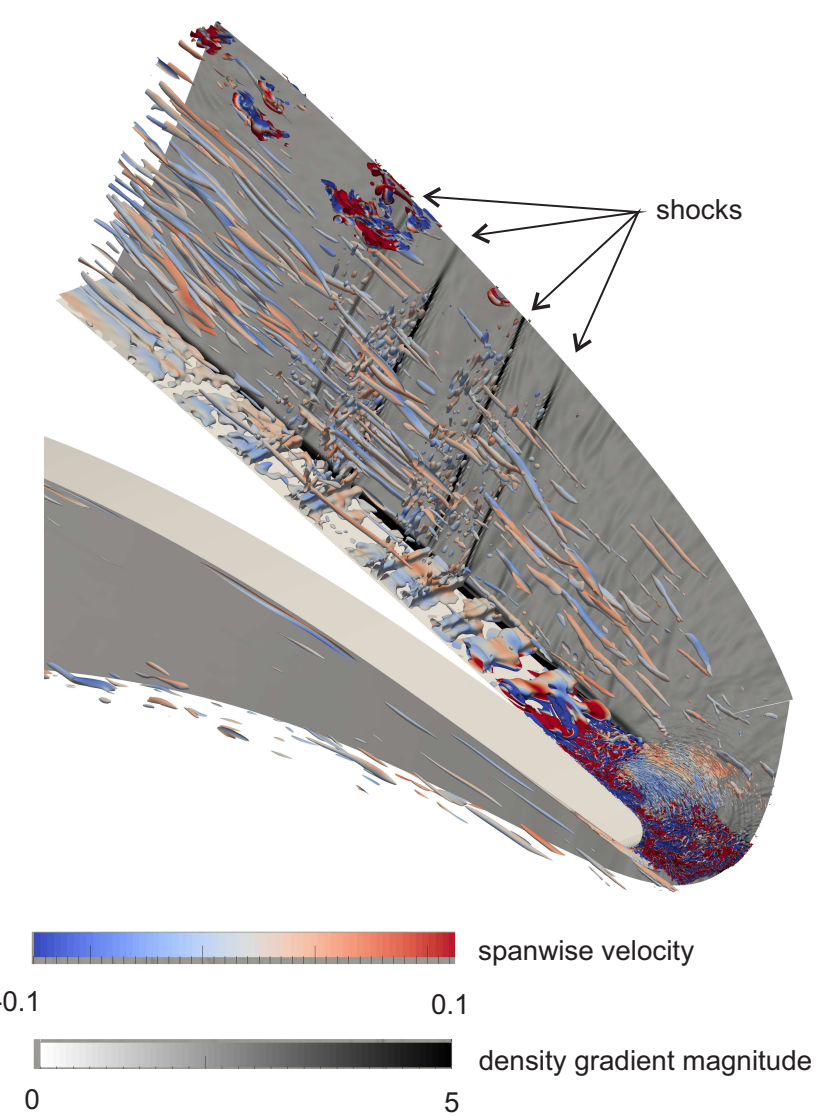

FIGURE 15. Iso-surfaces of Q-criterion $=50$ coloured by spanwise velocity in the region of transition

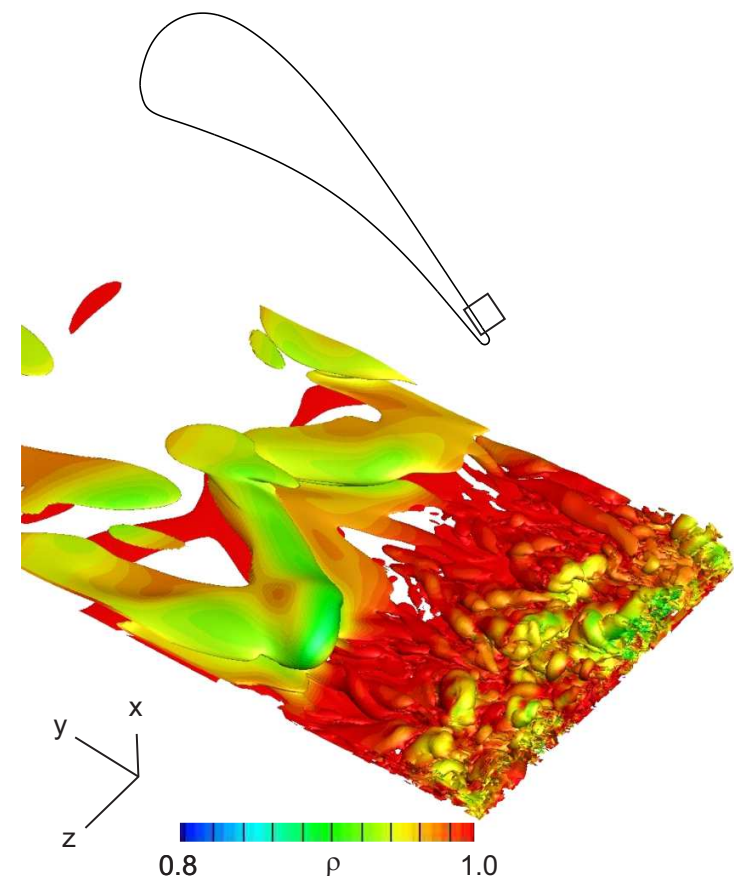

FIGURE 16. Iso-surfaces of vorticity magnitude $=1000$ coloured by density within transition region 


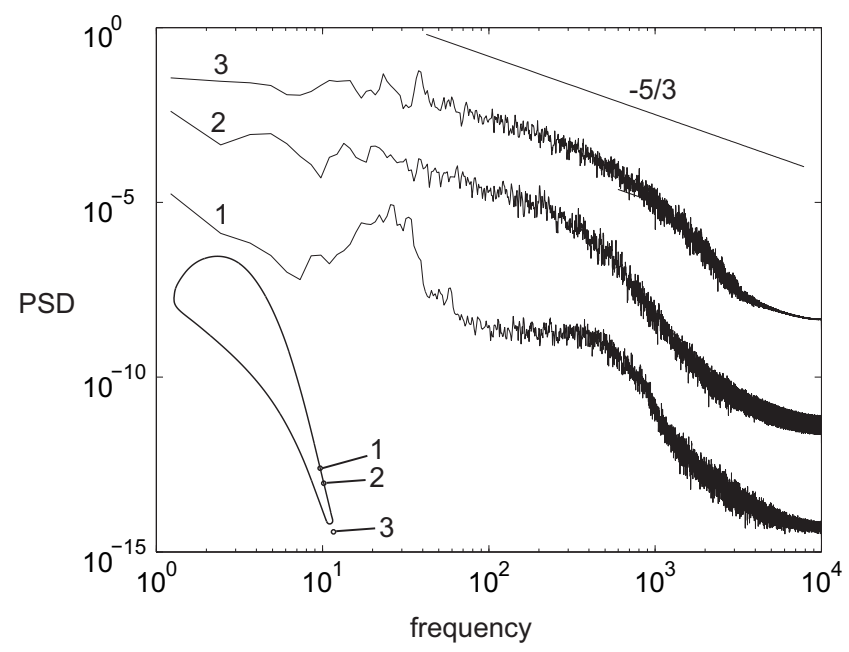

FIGURE 17. Power spectral density at two near-wall monitor points $(y+\approx 1)$ and a monitor point within the wake

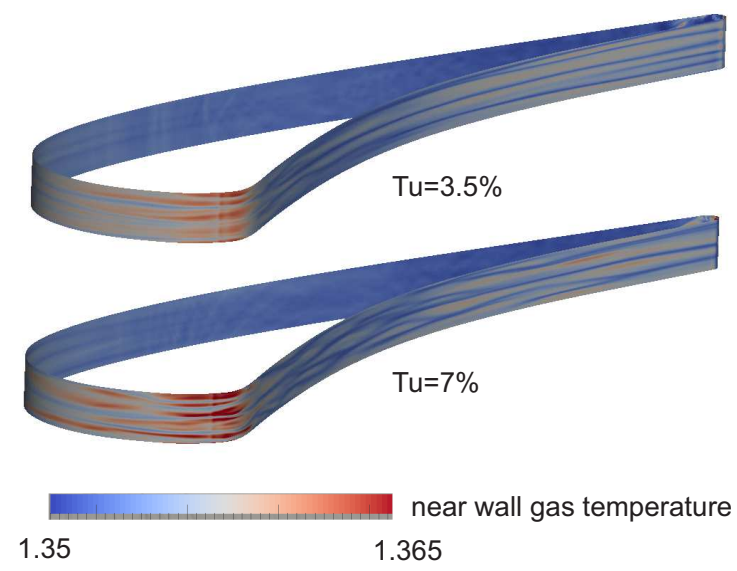

FIGURE 18. Instantaneous snapshot showing contours of gas temperature at the first grid point away from the wall

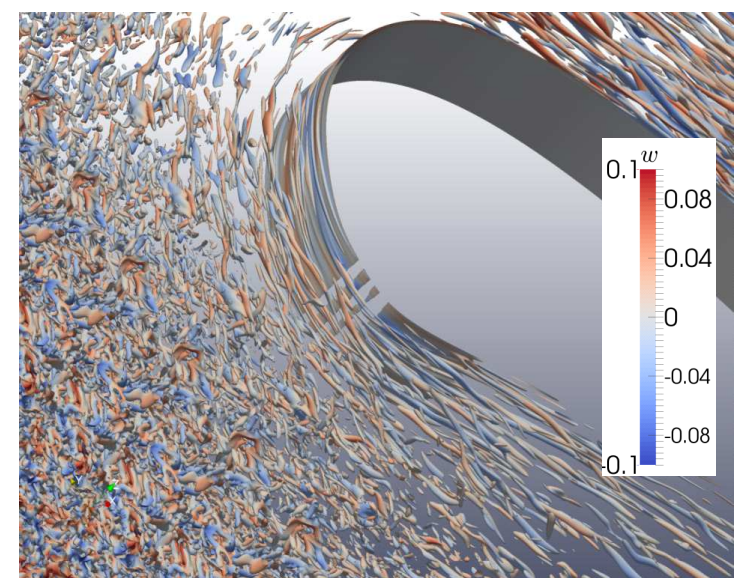

FIGURE 19. Iso-surfaces of Q-criterion $=25$ coloured by spanwise velocity near the leading-edge 


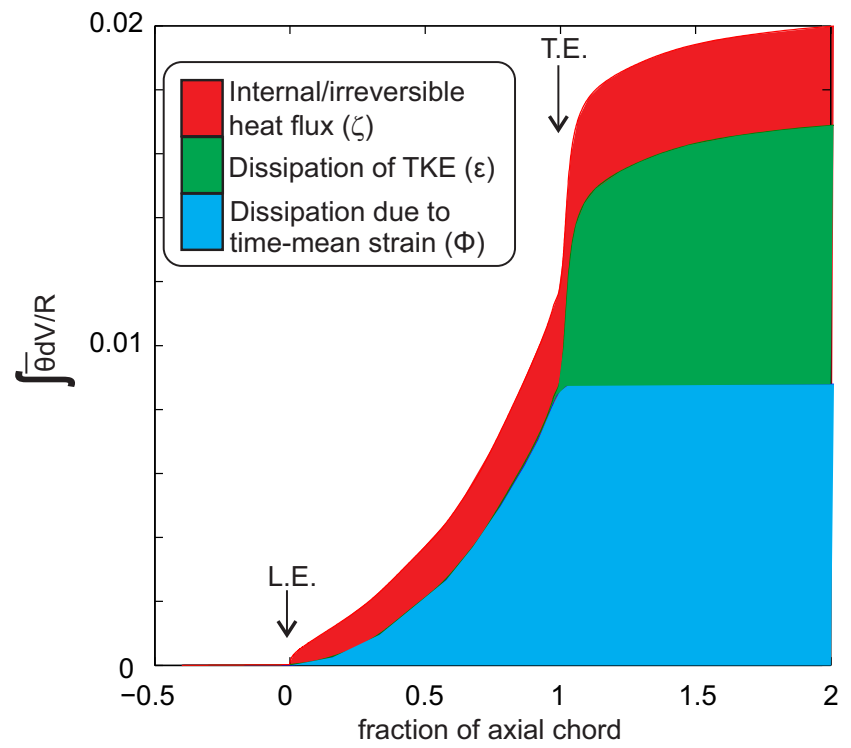

FIGURE 20. Rise in irreversible entropy through the vane passage and downstream 